Recepción: 10 / 01 / 2018

Aceptación: 09 / 02 / 2018

Publicación: 21 / 03 / 2018
Ciencias económicas y empresariales

Artículo de Revisión

\title{
Cultura tributaria y su incidencia en el Impuesto sobre la Renta
}

Tax culture and its incidence on Income Tax

\section{Cultura tributária e sua incidência no imposto de renda}

\author{
Claudia I. Melville-Pincay I \\ clingridmelville@hotmail.com
}

Correspondencia: clingridmelville@ hotmail.com

I Docente de la Universidad Técnica de Esmeraldas, Esmeraldas, Ecuador. 


\section{Resumen}

La propiedad ha de ser la primera variable que define las relaciones humanas modernas. En el ámbito tributario, es precisamente en tema de la necesidad de cumplir en donde se suscitan la mayor inconveniencia para la recaudación de impuestos sobre rentas. Debido a que un Estado primeramente es justo se debe tratar a cada miembro de la sociedad de manera equitativa. No obstante, en el andar de estos temas se hace evidente las distintas formas de adaptación o excusa que pretenden asumir un porcentaje de la población para evitar esta obligación. Por ello pretendemos repasar y reforzar los conocimientos, aportes y comentarios que acompañan a la situación actual de una sociedad cada día más civilizada, pero queda la incógnita a responder si, a estas alturas la cultura tributaria se ha fortalecido o por el contrario se ha deteriorado centrándonos en la relación que ésta mantiene con los niveles de recaudación. Se plantea en esta investigación sustentar teóricamente de qué manera una conducta social influye en la cuantificación de la acumulación de capital, se cree en la hipótesis que a medida que el ciudadano fortalezca su conciencia en las obligaciones tributarias impactará positivamente en el éxito de la recaudación. Las variables que siguen influyendo en los niveles de recaudación de impuesto sobre la renta, siguen siendo las mismas, aun mas, podemos asegurar que es en la cultura y conciencia tributaria en donde recae el mayor peso para el éxito de las políticas de Estado a nivel fiscal.

Palabras Clave: tributo; cultura; recaudación; evasión; conciencia.

\section{Abstract}

Property must be the first variable that defines modern human relations. In the tax field, it is precisely the issue of the need to comply where the greatest inconvenience for the collection of income taxes arises. Because a State is first fair, each member of society must be treated equitably. However, in the course of these issues it becomes evident the different forms of adaptation or excuse that a percentage of the population intends to take to avoid this obligation. Therefore we intend to review and reinforce the knowledge, contributions and comments that accompany the current situation of a society more and more civilized, but the question remains to answer if, at this point the tax culture has been strengthened or on the contrary has deteriorated focusing on the relationship that this maintains with the collection levels. In this research, it is 
argued theoretically in which way a social behavior influences the quantification of capital accumulation, it is believed in the hypothesis that as the citizen strengthens his conscience in the tax obligations he will have a positive impact on the success of the collection. The variables that continue to influence the levels of collection of income tax, remain the same, even more, we can assure that it is in the culture and tax conscience where the greatest weight for the success of State policies lies. fiscal.

Keywords: tribute; culture; collection; evasion; awareness.

\section{Resumo}

A propriedade deve ser a primeira variável que define as relações humanas modernas. No campo tributário, é justamente a questão da necessidade de cumprir onde surge o maior inconveniente para a cobrança de imposto de renda. Porque um Estado é justo em primeiro lugar, cada membro da sociedade deve ser tratado equitativamente. No entanto, no decorrer dessas questões, torna-se evidente as diferentes formas de adaptação ou desculpa que uma porcentagem da população pretende tomar para evitar essa obrigação. Por isso temos a intenção de rever e reforçar o conhecimento, contribuições e observações que acompanham a situação atual de uma sociedade cada vez mais civilizada, mas é desconhecido para responder se, neste ponto, a cultura fiscal foi reforçada ou de outra forma se deteriorou enfocando a relação que isso mantém com os níveis de coleta. Surge neste apoio à investigação teoricamente como comportamento social afeta a quantificação da acumulação de capital, acredita-se na hipótese de que, como cidadãos reforçar a sua consciência das obrigações tributárias irá impactar positivamente o sucesso da coleção. As variáveis que continuam a influenciar os níveis de arrecadação do imposto de renda, permanecem as mesmas, ainda mais, podemos assegurar que é na cultura e na consciência tributária onde reside o maior peso para o sucesso das políticas de Estado. fiscal.

Palavras chave: tributo; cultura; coleção evasão consciência. 


\section{Introducción}

En la evolución del hombre está el desarrollo de la sociedad. La propiedad ha de ser la primera variable que define las relaciones humanas modernas. Dicha propiedad no necesariamente se refiere a la acumulación de bienes sino, incluso a la potestad sobrenatural intangible del derecho divino y relación directa con los dioses.

Para el investigador social es fácil realizar un conducto directo entre los dioses de aquel olimpo y las relaciones sociales de la plebe y la sangre azul. Sin embargo, no es la intensión de esta investigación asumir que cada lector posee todos los conocimientos, sino brindar una propuesta al conocimiento propio y colectivo.

A todas estas remontamos en los romanos, pues es allí en donde se origina los fundamentos del derecho que rige la ciencia y la sociedad que nos arropa. El derecho romano y aquellos primeros mandamientos marcan el inicio de unas normas que organizan ciertas conductas, no digamos naturales del ser humano, pero si conductuales del ser social.

Primitivamente, los hombres y mujeres transformaron su vida nómada en una vida local sedentaria, con este cambio se hace necesario el trabajo de la tierra para cosechar alimentos y la delimitación de espacios. A medida que esto ocurre, nace la propiedad, también las obligaciones, la legislación y para ahorrarnos un poco la historia nace la necesidad de desarrollar mecanismos para generar deberes ciudadanos, exigir derechos ciudadanos y brindar bienes colectivos.

Entre esos derechos conquistados para el privado también surge el derecho público, el derecho de una nación por sobre sus ciudadanos y más allá de justificar esa necesidad, se busca conocer el rol personal y público que juega dentro del nombrado mundo tributario.

\section{Entre los conceptos encontrados conseguimos:}

"El legislador, juzga que determinados hechos de la vida social son reveladores de capacidad contributiva y los justifica, elevándolos a presupuesto de hecho de los tributos y atribuyéndoles, consiguientemente, el efecto jurídico de hacer surgir a cargo de ciertas personas una obligación tributaria." (Palao Taboada, 2009)

En este campo se conjugan el Derecho, la Contabilidad y la Economía en lo macro y micro puesto que de allí deriva la obligación del legislado, el registro de los ingresos de la nación y por 
ende su inversión. Todo este proceso se crea y se perfecciona con el fin de evitar crear incentivos corruptos para evadir obligaciones.

En el ámbito tributario, es precisamente en tema de la necesidad de cumplir en donde se suscitan la mayor inconveniencia para la recaudación de impuestos sobre rentas. Debido a que un Estado primeramente es justo se debe tratar a cada miembro de la sociedad de manera equitativa. No obstante, en el andar de estos temas se hace evidente las distintas formas de adaptación o excusa que pretenden asumir un porcentaje de la población para evitar esta obligación.

Rodríguez Lobato (2002), explica que, "la contribución es un concepto genérico que engloba a todo lo que auxilia a sufragar los gastos del Estado. Este género se divide en contribuciones forzadas y contribuciones voluntarias". Las primeras, llamadas también exacciones, son las que fija la ley a cargo de los particulares que se adecuen a la hipótesis normativa prevista en la propia ley y las segundas, las voluntarias serían las que derivan de un acuerdo de voluntades entre el Estado y el particular, o de una declaración unilateral de voluntad del particular que implica un acto de liberalidad. (Arroyo Grant, Espinosa Mejía, \& Amezcua García, 2014)

Por ello pretendemos repasar y reforzar los conocimientos, aportes y comentarios que acompañan a la situación actual de una sociedad cada día más civilizada, pero queda la incógnita a responder si, a estas alturas de nuestros Estados la cultura tributaria se ha fortalecido o por el contrario se ha deteriorado centrándonos en la relación que ésta mantiene con los niveles de recaudación. Aún más, quizá nos podamos encontrar con que los mecanismos de recaudación del Estado y su estructura legislativa y judicial han logrado independizar la variable dependiente de la conciencia social para lograr una recaudación suficiente, todo esto basado en una revisión de literaturas.

\section{Metodología}

Las ciencias sociales se consideran un punto de encuentro para el enlace perfecto para desarrollar una mezcla de investigación cualitativa e investigación cuantitativa, pues en ella se centra el enigma de cómo medir una variable cultural en donde pareciera que la subjetividad juega el principal rol. 
Resulta que, es en las ciencias sociales como Economía, Administración, Sociología o Contaduría Pública en donde existe la necesidad de enlazar las ciencias exactas en un proceso científico en cuya inexactitud se encuentra la verdadera ciencia.

A manera de explicar esta disyuntiva, se plantea en esta investigación sustentar teóricamente de qué manera una conducta social influye en la cuantificación de la acumulación de capital, se cree en la hipótesis que a medida que el ciudadano fortalezca su conciencia en las obligaciones tributarias impactará positivamente en el éxito de la recaudación.

Por supuesto, primeramente, se hace obligatorio identificar que variables influyen en esa cultura tributaria, para luego comprobar o desechar la hipótesis de manera teóricamente cuantitativa.

Para alcanzar el objetivo nos centramos en la revisión bibliográfica de las publicaciones científicas recientes o referenciales, de ellas desprender la relación teórica existente entre la cultura tributaria y la recaudación de Impuestos sobre la Renta (ISLR), resultando en un fichaje de hallazgos pertinentes para tal objetivo y concluyendo en la relación resultante.

\section{Resultados}

Podemos comenzar esta exposición de trabajos de investigación con una exposición de los fines tributarios amparados en la Constitución de la República del Ecuador en donde demanda que:

"La soberanía radica en el pueblo, cuya voluntad es el fundamento de la autoridad, y se ejerce a través de los órganos del poder público y de las formas de participación directa previstas en la Constitución, por tanto, se debe planificar el desarrollo nacional, erradicar la pobreza, promover el desarrollo sustentable y la redistribución equitativa de los recursos y la riqueza, para acceder al buen vivir. El régimen tributario se regirá por los principios de generalidad, progresividad, eficiencia, simplicidad administrativa, irretroactividad, equidad, transparencia y suficiencia recaudatoria. Se priorizarán los impuestos directos y progresivos. La política tributaria promoverá la redistribución y estimulará el empleo, la producción de bienes y servicios, y conductas ecológicas, sociales y económicas responsables. Los tributos, además de ser medios para recaudar ingresos públicos, servirán como instrumento de política económica general, estimulando la inversión, la reinversión, el ahorro y su destino hacia los fines productivos y de desarrollo 
nacional; atenderán a las exigencias de estabilidad y progreso sociales y procurarán una mejor distribución de la renta nacional. (Quintanilla, 2012)

En teoría, esta sería una explicación completa y compleja de cuál es la concientización que debe promover el Estado para justificar vehementemente la imposición de tributos. En esta exposición no solo demanda una conciencia del ciudadano sino también una conciencia de Estado en cuanto esos recursos deben ser destinados a un fin específico.

Para una región como Latinoamérica, tomar como punto de partida el Estado Ecuatoriano hay que considerar las características de la sociedad que la define, considerar nivel de empleo, PIB per cápita, desigualdad social y participación del Estado en las relaciones económicas, estas variables al verlas en un espectro amplio pueden parecerse por lo que teóricamente se ajusta para la región y puede calar el discurso entre los ciudadanos.

Caso contrario puede suceder en otras latitudes en donde la cultura, la sociedad y el resultado de las políticas gubernamentales han sido distintos a las latinoamericanas.

Se hace necesario ir descubriendo de que se trata la cultura tributaria en el presente, pero por sobre todas las necesidades se trata de estudiar como calar una sana cultura en la sociedad.

“parte de la base para formar una cultura tributaria está en la concientización, la cual va más allá de una simple acción, implica todo un proceso que va desde la sensibilización hasta la reflexión, que logre que las personas seamos capaces de dimensionar el potencial que tenemos para transformar nuestro entorno y realidad al formar parte de ella... Concientización fiscal Si al momento de ingresar a la esfera fiscal, se lograra que desde niños tuviésemos una conciencia de responsabilidad, compromiso, legalidad y solidaridad, la conducta fiscal sería diferente, pues no habría vacíos de conocimientos, compromiso y responsabilidad para cumplir con las obligaciones fiscales, que se han venido gestando en las últimas décadas en la que la ausencia del reforzamiento de valores por parte del gobierno ha ido en contra del beneficio de la patria, por lo que se requiere del compromiso de todos los actores de manera tripartita entre gobierno, instituciones de educación y la sociedad para que impacten como pilar de la cultura tributaria”. (Arroyo Grant, Espinosa Mejía, \& Amezcua García, 2014) 
En la vida cotidiana de la ciudadanía, se forman personas con valores que son capaces de comprender las leyes y normas que determinan la vida social, lo que facilita el cumplimiento de los deberes ciudadanos principalmente enfocados en la tributación, lo cual contribuiría en parte a tener respeto por la patria y por todo aquello que simboliza la identidad nacional, permitiendo fortalecer los valores cívicos que hemos venido perdiendo en las últimas décadas como lo es del dominio público, pero que sin embargo, consideramos desde nuestro humilde punto de vista que son los cimientos para construir a la identidad étnico-cultura de cualquier nación... El fenómeno de la legitimidad de la coerción, en el marco del Estado moderno, consiste en la aceptación de la misma como necesaria por parte de los ciudadanos, situación posible sólo en la medida en que comparten los objetivos que la guían y los valores y normas que busca preservar. En relación con el problema de la legitimidad de las acciones de fiscalización, proponemos como hipótesis que el grado de legitimidad que puedan obtener las acciones de la administración tributaria es el reverso del grado de ilegitimidad que corresponda al incumplimiento tributario, la evasión y el contrabando. Si bien es imposible establecer relaciones mecánicas en el ámbito de las representaciones y valoraciones colectivas, resulta sensato sostener que un mayor rechazo social hacia el incumplimiento tributario constituye un terreno propicio para las actividades de fiscalización”. (Cortázar, 2000)

La evasión fiscal es el principal inconveniente, y más allá de ello destacan los incentivos para evadir. Es por ello que se busca apelar a los sentidos, a la conciencia, a un motor intangible que parece seguir demostrando que tiene incidencia en el éxito de las políticas fiscales.

"La evasión impositiva ha sido, a lo largo de las últimas décadas una de las características centrales de los países en desarrollo. En este hecho inciden fenómenos de distinta naturaleza, desde cuestiones de índole sociocultural hasta factores más complejos de atraso tecnológico y deficiente administración tributaria. Al mismo tiempo, estos países tienen menor ingreso per cápita y sus sociedades acostumbran a mostrar importantes desigualdades entre los distintos estratos de ingreso. Esto lleva a que la política fiscal, y por ende la política tributaria, tengan un papel significativo en cuestiones de equidad y distribución de la renta". (Chelala \& Giarrizzo, 2014) 
"Los países con altos niveles de recaudación normalmente tienen una mayor fortaleza institucional (Besley y Persson, 2009), lo que les permite presionar a sus ciudadanos para que cumplan con sus obligaciones. Así, es probable que se determine una asociación positiva con la moral tributaria. Del mismo modo puede ocurrir cuando la tasa máxima del isrp es elevada, pues ello favorece la progresividad del impuesto y transmite la idea de que el sistema es justo (quienes más tienen, más pagan). En síntesis, los signos de estas dos variables son ambiguos, pues hay argumentos que se contraponen.” (Castañeda Rodríguez, 2015)

"El efecto multiplicador está sustentado en el gasto público y los ingresos, el cual permite generar el manejo financiero público, en este contexto, los multiplicadores fiscales intentan calcular los efectos dinámicos de la política físcal sobre la economía utilizando modelos complejos como los estructurales vectoriales auto regresivo (SVAR) o los dinámicos estocásticos de equilibrio general (DSGE). Es decir, miden los efectos que un tipo y un tamaño determinado de expansión o de contracción fiscal pueden tener sobre el producto y la tarea de crecimiento de un país." (Castillo, 2010) (Gamboa, Hurtado, \& Ortiz, 2017)

"Para especificar la distribución de la carga tributaria se han planteado diversos tópicos: privilegiar el impuesto a los ingresos con tasas progresivas; impuestos al valor agregado (impuesto a la venta de bienes y servicios), con tasas reducidas o tipo cero para bienes de primera necesidad; seguridad social basada en sistemas de reparto; establecimiento de impuesto a rentas de capital, patrimonio y herencia; mínima cantidad de beneficios tributarios en el esquema tributario de ingresos y rentas de capital; aplicar aranceles e impuestos especiales para proteger empresas incipientes o pequeñas, resguardar recursos naturales, establecer regímenes más benéficos para actividades económicas desarrolladas por personas pobres, etc. Por su parte, sería contrario a la justicia tributaria: el establecimiento de impuestos planos; impuestos a la renta iguales para las personas; impuestos al valor agregado sin exenciones; sistema previsional de capitalización individual; regímenes de baja imposición a las rentas de capital; ausencia de tributación al patrimonio y la herencia; exenciones y reducciones disponibles solo para grandes patrimonios, etc.

Por otro lado, la voz "justicia" expresa una cualidad de los tributos (como adjetivo calificativo), de todos o alguno en particular, o del sistema tributario en su conjunto. De ahí que es posible 
construir la expresión "justicia tributaria”, como justicia referida al ámbito de la imposición de gravámenes. Con todo, como puede observarse, la categoría "justicia tributaria" puede ser entendida de múltiples modos, con múltiples contenidos y alcances. Esta polisemia se puede limitar a través del establecimiento de principios y reglas, entendidas ellas como normas jurídicas. (Masbernat, 2014)

"Si bien el Impuesto Sobre la Renta, por su parte, fue incorporado en los sistemas tributarios de América Latina con mucha anterioridad respecto al IVA 24, la importancia relativa de este tributo recién tomó un primer gran impulso en las décadas del 50 y 60 con el surgimiento del enfoque redistributivo de la tributación y la política fiscal (Kaldor, 1962). Según Cornia et al. (2011), en varios países de América Latina la imposición directa alcanzó una participación relativa en torno al 30\% de los ingresos tributarios, aun cuando encontrara grandes obstáculos iniciales dados por la estructura predominantemente rural e informal de sus economías, la elevada desigualdad de ingresos y una baja calidad institucional y administrativa. Luego, las reformas neoliberales de los años 70 y 80 corrieron a este impuesto del centro de la escena tributaria y fiscal. Durante esos años tomó fuerza, entre la mayoría de los analistas y autoridades de los países, la creencia de que las elevadas tasas impositivas, además de impopulares, no sólo desalentaban la actividad económica, sino que tampoco eran efectivas para mejorar la distribución del ingreso y la riqueza (Bird y Zolt, 2005). Esto condujo a los países a disminuir la cantidad y los niveles de las alícuotas legales del ISR para personas naturales. En sentido contrario a lo observado con la tasa general del IVA, la alícuota marginal máxima promedio cayó de un $49,5 \%$ a 29,1\% entre 1980 y 2000, continuando un leve descenso hasta un valor de 28,1\% en 2011”. (Gómez \& Morán, 2013)

\section{Conclusiones}

Creando el hilo conductual hilvanado en los resultados vamos concluyendo que "La cultura tributaria debe entenderse como una forma de vida en donde tengamos conciencia del papel que jugamos dentro de la sociedad, conjugando manifestaciones donde se cultiven los valores, promoviendo actitudes y comportamientos orientados al cumplimiento de nuestras obligaciones tributarias". (Cortázar, 2000). 
Las variables que siguen influyendo en los niveles de recaudación de impuesto sobre la renta, siguen siendo las mismas, aun más, podemos asegurar que es en la cultura y conciencia tributaria en donde recae el mayor peso para el éxito de las políticas de Estado a nivel fiscal.

Es menester de la estructura oficial recaudadora promover la concientización del ciudadano, que entienda y asuma el compromiso de cumplir con sus obligaciones como contribuyente y también es obligatoria una gestión visible de inversión social que genere confianza en el contribuyente para sentirse parte de una labor social en donde se cumpla el efecto multiplicador que en la medida en que el ISLR sea aplicado de forma equitativa como mecanismo de distribución de renta, recibirá para el colectivo una cuantía equivalente de beneficios sociales, administrados por el Estado pero financiado por los contribuyentes.

\section{Referencias Bibliográficas}

Arroyo Grant, M. G., Espinosa Mejía, F., \& Amezcua García, E. (2014). La concientización fiscal de los contribuyentes, como base para formar cultura tributaria en México. Ciencia Administrativa, 144-150.

Castañeda Rodríguez, V. M. (2015). La moral tributaria en América Latina y la corrupción como uno de sus determinantes. Revista mexicana de Ciencias Políticas y sociales, 103-132.

Chelala, S., \& Giarrizzo, V. (2014). Evasión de impuestos en Argentina: un análisis experimental de la eficacia de premios y castigos al contribuyente. Finanzas, política y Economía, 269-286.

Cortázar, J. (2000). Estrategias educativas para el desarrollo de una "cultura Tributaria" en América Latina. Reforma y Democracia, http://siare.clad.org/revistas/0037901.pdf.

Gamboa, J., Hurtado, J., \& Ortiz, G. (2017). Gestión de la política fiscal para fortalecer la cultura tributaria en Ecuador. Publicando, 448-461.

Gómez, J., \& Morán, D. (2013). Política Tributaria en América Latina: agenda para una segunda generación. Santiago de Chile: Publicación de las naciones Unidas.

Masbernat, P. (2014). Justicia y sistema tributario. Una mirada desde la perspectiva inglesa. Dikaion, 135-169. 
Palao Taboada, C. (2009). La aplicación de las normas tributarias y la elusión fiscal. España: Lex Nova, S.A.

Quintanilla, J. (2012). La universidad en la cultura tributaria. Retos, 106-114. 